\title{
Liquid crystal emulsion micro-droplet WGM resonators
}

\author{
Jan Ježek $^{1 *}$, Zdeněk Pilát ${ }^{1}$, Oto Brzobohatý ${ }^{1}$, Alexandr Jonášs ${ }^{2,3}$, Mehdi Aas ${ }^{2}$, Alper Kiraz ${ }^{2}$, \\ Pavel Zemánek ${ }^{1}$ \\ 1) ASCR, Institute of Scientific Instruments, Královopolská 147, 61264 Brno, \\ Czech Republic \\ ${ }^{2)}$ Department of Physics, Koç University, Rumelifeneri Yolu, Sariyer, 34450 Istanbul, \\ Turkey \\ ${ }^{3)}$ Department of Physics, Istanbul Technical University, Maslak, 34469 Istanbul, Turkey \\ *jezek@isibrno.cz; phone +420 541514 524; fax +420 541514 402; www.isibrno.cz/omitec
}

\begin{abstract}
We introduce tunable optofluidic microlasers based on optically stretched or thermally modified, dye-doped emulsion droplets of liquid crystals (LC) confined in a dual-beam optical trap. Droplets were created in microfluidic chips or by shaking. Optically trapped microdroplets emulsified in water and stained with fluorescent dye act as an active ultrahigh-Q optical resonant cavity hosting whispering gallery modes (WGMs). Tuning of the laser emission wavelength was achieved by a controlled deformation of the droplet shape using light-induced forces generated by dual-beam optical trap and by thermal changing of the order in the LC.
\end{abstract}

Keywords: optical trapping, microfluidic chips, liquid crystals, whispering gallery modes, droplets

\section{INTRODUCTION}

Various applications in physics, chemistry, biology, medicine, etc. are possible and convenient to carry out in small volumes, directly in the field of view of the optical microscope, and with high precision of the sample dosing. Downsizing brings many advantages such as reducing the consumption of reagents for chemical analysis. It is possible to observe a range of reactions in parallel, to shorten reaction times, maintain stable sample temperature, low reagent volumes imply increased safety, etc. These trends lead to complex microfluidic systems, such as "lab-on-a-chip" [1]. Microfluidic chips consist of channel systems, chambers and various control and management elements (pumps, mixers, valves, etc. $[2,3]$ ) connected to generally complicated structures with dimensions in the order of units to hundreds of micrometers. The possibility of precise fluid flow control in such scale opens a wide field for possible inclusion of optical micromanipulation techniques, optical sorting [4], laser ablation, microspectroscopy, or microphotopolymerization [5, 6].

Excitation of whispering gallery modes (WGM) lasing in fluorescently labeled droplets of water emulsified in low refractive index oil in microfluidic channel was reported previously [7]. Droplet based microresonators from cholesteric (chiral) liquid crystals (LC) were fabricated in microfluidic channel and sorted according to their chirality by their interaction with laser beam [8]. WGM lasing of nematic LC droplets in microchannel was used for chemical sensing of surfactant concentration [9]. Spectral characteristics of WGM lasing in cholesteric LC droplets were shown as well [10]. Various principles of spectral tuning of WGM lasing were employed for microresonators based on LC, oil, or water droplets [11-13]. Optomechanical characteristics of solid chiral microresonators dependent on laser beam polarization were also studied [14].

\section{MATERIALS AND METHODS}

We devised tunable optofluidic microlasers based on optically stretched, dye-doped emulsion droplets of nematic liquid crystals (LC) confined in an optical trap. Optically trapped microdroplets of LC emulsified in water and stained with fluorescent dye act as active ultrahigh-Q optical resonant cavities hosting whispering gallery modes (WGM) which enable dye lasing with low threshold pump powers. In order to achieve tunable dye lasing, the droplets were trapped in a single beam optical tweezers, pumped with a pulsed green laser beam and simultaneously electrically heated or stretched by a dual-beam laser trap. Experimental setup for the observation of tunable lasing from optically trapped and stretched or heated emulsion microdroplets is shown in figure 1.

19th Polish-Slovak-Czech Optical Conference on Wave and Quantum Aspects of Contemporary Optics, edited by Agnieszka Popiołek-Masajada, Wacław Urbańczyk, Proc. of SPIE Vol. 9441, 944108 (C) 2014 SPIE · CCC code: 0277-786X/14/\$18 - doi: 10.1117/12.2176025 
Studied oil-in-water emulsions were contained within home-made PDMS microfluidic chips featuring a single channel with square cross-section of $160 \times 160 \mu \mathrm{m}$. These chips supported either a meander of a resistor wire for heating the channel contents, see figure 2, or two multimode optical fibers with $50 \mu \mathrm{m}$ core and $125 \mu \mathrm{m}$ cladding diameters, see figure 3B. The faces of the fibers were parallel to the opposite side walls of the channel and their optical axes were precisely aligned to form the dual-beam trap. The microfluidic channel had one inlet and one outlet with a syringe connected to the inlet via tubing to inject previously prepared microdroplet emulsions and washing solutions. Detailed manufacturing process for the chips is given in the next subsection. In order to observe the droplets, excite and collect the fluorescence emission from the droplets, and implement single beam optical trap, PDMS chips were mounted on an xyz stage of an inverted optical microscope equipped with a water immersion objective (NA=1.2, 60x; Nikon). Laser light source for the dual-beam trapping and optical stretching was a $1070 \mathrm{~nm}$ Ytterbium fiber laser (YLM-10-LP-SC; IPG Photonics) with $10 \mathrm{~W}$ maximum output power.

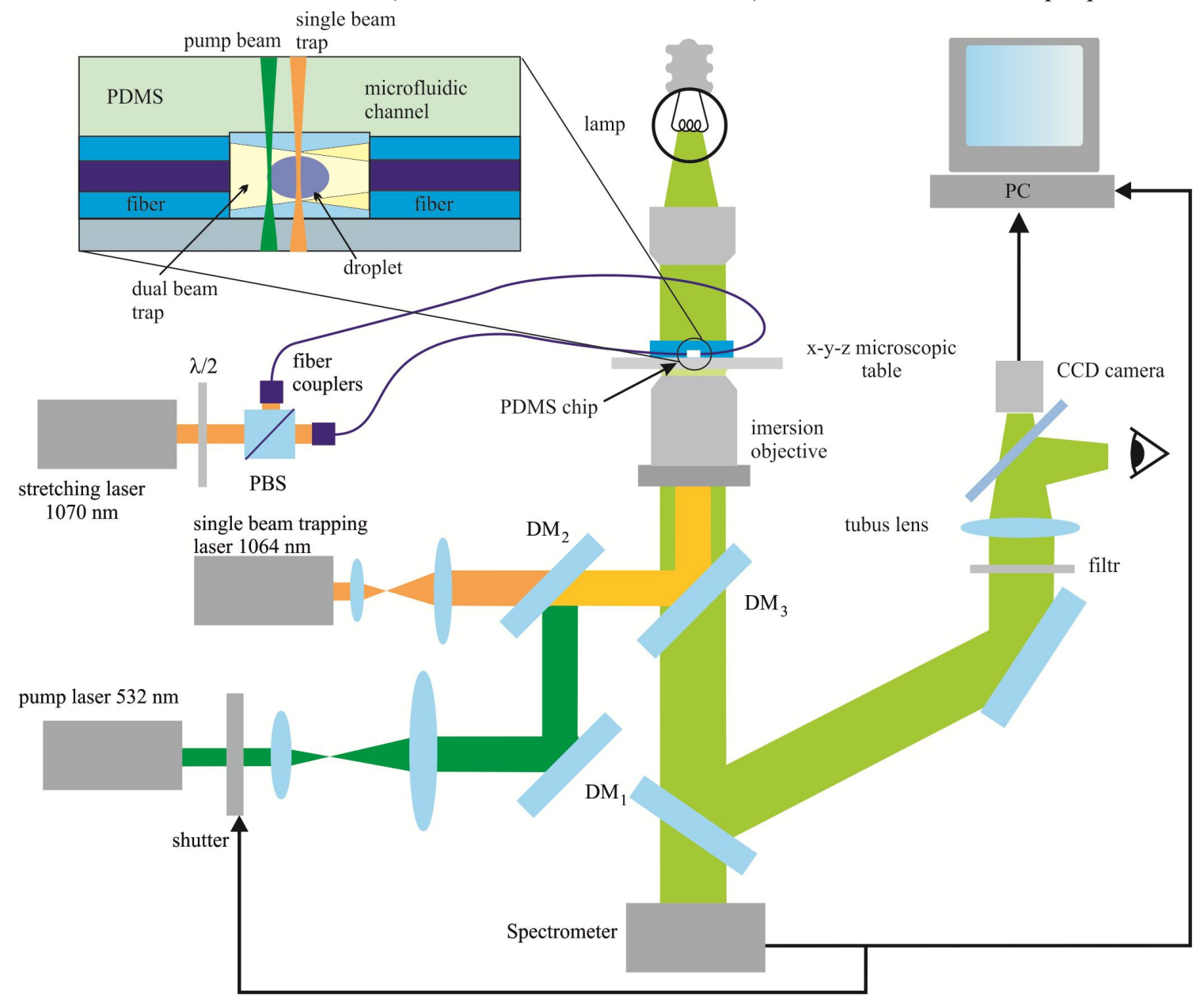

Figure 1: Experimental setup: Setup was mounted on an inverted optical microscope equipped with a water immersion objective. Trapping laser (Nd:YAG, $1064 \mathrm{~nm}$ ) and pump laser (frequency-doubling Nd-YVO4, $532 \mathrm{~nm}$ ) were implemented into microscope through fluorescence port. Inset: PDMS microfluidic chip featured one straight channel with square crosssection of 160x160 $\mu \mathrm{m}$ and two precisely aligned optical fibers (multimode fiber with core diameters of $50 \mu \mathrm{m}$ ) in the middle which were used for droplet deformation by optical stretching (fiber laser IPG $1070 \mathrm{~nm}$ ).

Its output beam was directed to a $\lambda / 2$ waveplate (WP1) mounted on a stepper-motor driven rotary stage and a polarizing beam splitter (PBS1) which together served to adjust the total stretching power coupled into the fibers. Following PBS1, another polarizing beam splitter PBS2 was used to divide the beam into two parts, with $\lambda / 2$ waveplate WP2 mounted on a manual rotary stage adjusting the division ratio of the total beam power between the fibers. Subsequently, both beams were coupled to respective single-mode patch cables (P5-980A-PCAPC-1; Thorlabs) using compact fiber couplers (PAF-X-11-C; Thorlabs). The output ports of the patch cables were then 
connected to the free ends of two multimode fibers embedded in the microfluidic chip. In order to measure the total actual power coupled to the fibers, a glass plate with an angle of $45^{\circ}$ relative to the laser beam was inserted in the beam path after PBS1; this plate reflected a small fraction of the total power (about $5 \%$ ) towards a power meter. Therefore, the measured power fraction was calibrated by measuring the total output power from the bare fiber ends before inserting them into the chip.

We were trapping nematic LC droplets with radial and bipolar arrangement. We have used 5-CB (4-cyano-4'pentylbiphenyl, Sigma-Aldrich), either pure, or mixed with 1:1 (w/w) with 8-OCB (4-octyloxy-4'cyanobiphenyl, Sigma-Aldrich) as the nematic LC substrate, doped with $0.1 \%$ (w/w) Nile Red fluorescent dye (9-diethylamino-5-benzo[ $\alpha]$ phenoxazinone, Sigma-Aldrich). We have used $4 \mathrm{mM}$ SDS (sodium dodecylsulfate, Sigma-Aldrich) solution for induction of the radial geometry. The emulsions of LC were prepared as follows:

\section{5 -CB or $8-\mathrm{OCB}+5-\mathrm{CB} 1: 1(\mathrm{w} / \mathrm{w}) \quad 100 \mathrm{mg}$ \\ Nile Red (or different fluorescent dye) $\quad 0.1 \mathrm{mg}$}

This mixture of $\mathrm{LC}$ and dye was heated to approx. $80^{\circ} \mathrm{C}$ and thoroughly mixed. Then the emulsion was prepared in the following manner:

$\mathrm{LC}+$ dye mixture $\quad 10 \mu \mathrm{l}$

$4 \mathrm{mM}$ SDS (radial drop.) or deionized water (bipolar drop.) $1 \mathrm{ml}$

The resulting two-phase mixture was shaken for several seconds by hand, until the droplets reached the desirable size, which was controlled by observation in optical microscope.

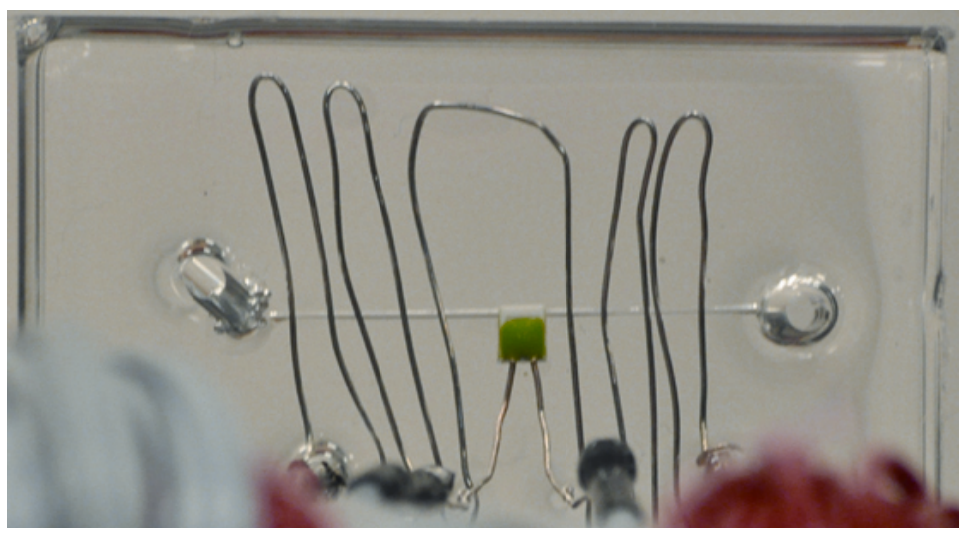

Figure 2: The heating chip. A channel with wide input ports is placed under a meander of a resistor wire. Near the center, this opens to a larger loop, inside which, thermistor is placed (green). The body of the chip is from PDMS. The input and sensing leads are in the forefront.

The droplets of LC prepared in the manner described above were subjected to trapping with increasing power in the dual beam trap (1064 $\mathrm{nm}$ laser). The stepwise increase in the dual beam power was from approx. $100 \mathrm{~mW}$ to approx. $1100 \mathrm{~mW}$ in the sample plane (total for both inputs), in 10 or 20 equal steps, followed by the same amount of steps for a gradual decrease to the base value. During this ramping and de-ramping, the laser pulses of $532 \mathrm{~nm}$ laser were used for excitation of WGM lasing modes in the LC droplet. The position of the droplet was stabilized by an auxiliary single beam trap centered between the two optical fibers of the dual beam trap (1070 $\mathrm{nm}$ laser). The excitation pulses were directed to the rim of the trapped droplet, either on the axis of the dual beam trap, or perpendicular to this axis, see figure 3A. 

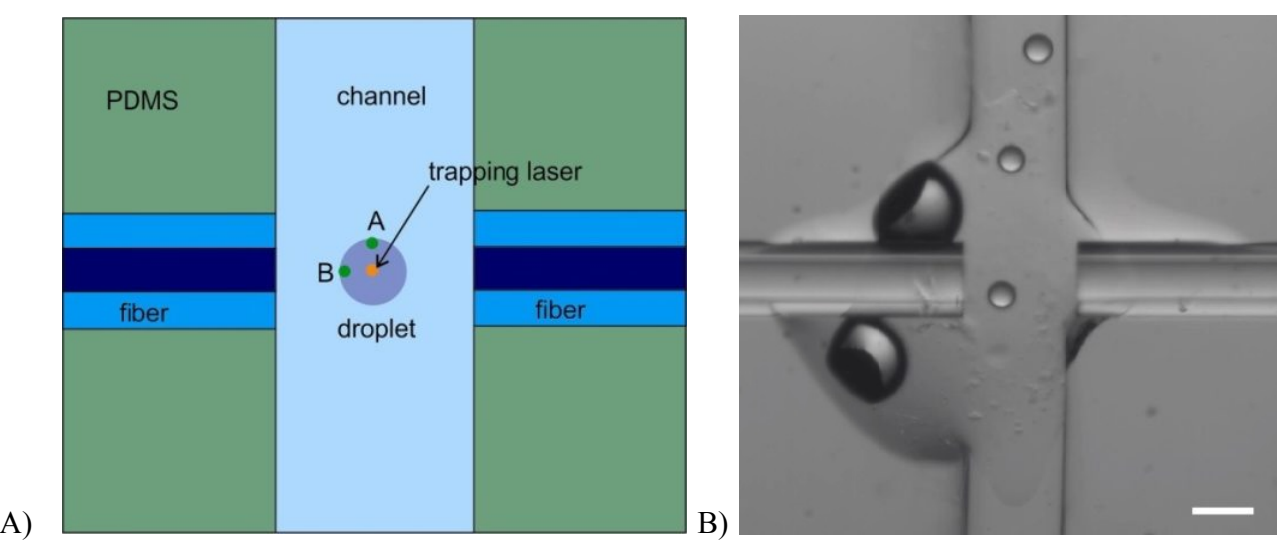

Figure 3: A) The schematized geometry of the emulsion LC droplet trapping experiment. The figure depicts the PDMS chip with optical fibers of the dual beam trap entering from the sides the microfluidic channel in the center. The droplet is trapped in the dual beam trap. The green point A on the droplet corresponds to the $532 \mathrm{~nm}$ pulsed WGM excitation "off axis", perpendicular to the axis of the dual beam trap, while the green point B corresponds to excitation "on axis", concomitant to the axis of the dual beam trap. The droplet is concurrently trapped with a single beam trapping laser in the center (orange point), in order to stabilize its position in the dual beam trap. B) The dual beam trapping setup with droplets injected in the channel between the optical fibers. Scale-bar: $100 \mu \mathrm{m}$.

In an alternative experiment in order to examine different temperature related properties of laser emission from dye doped LC droplets, we have used the heated microfluidic chip from PDMS and a resistor wire (figure 2). We have loaded the channel with prepared LC emulsion droplets, then connected the resistor wire to a source of regulated voltage and measured the temperature rise with an integrated thermistor. With the resistance of the wire about $3.5 \mathrm{ohms}$, we have operated the chip up to approx. $80^{\circ} \mathrm{C}$ at input voltage up to $3 \mathrm{~V}$. We have trapped the LC droplet in the auxiliary trap and excited the WGM by green laser pulses during a temperature sweep of approximately $10^{\circ} \mathrm{C}$, covering the transition temperature of $5-\mathrm{CB}$. With the temperature changes, the chip displayed a tendency to bend, so it was necessary to refocus during an observation of the trapped LC droplet. Below $10^{\circ} \mathrm{C}$ difference, this effect was not so pronounced, and the refocusing was not necessary.
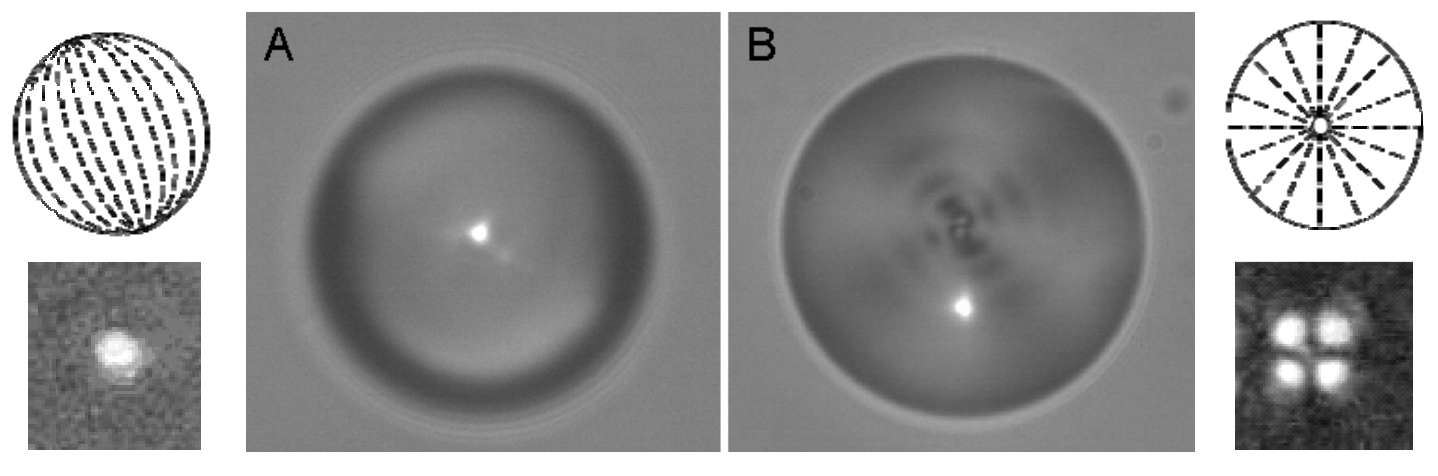

Figure 4: Optical trapping of bipolar (A) and radial (B) LC droplet in a single beam optical trap. Note that the radial droplet appears to be trapped half-way between the center and the edge of the droplet, while the bipolar droplet is trapped in the center. The diameter of the depicted droplets is around $50 \mu \mathrm{m}$. Small pictures show schematic depiction of the molecular director in a nematic LC and image from polarization microscope for the respective orientations of the molecular director of the LC in a droplet.

\section{RESULTS}

We have observed the trapping of radial and bipolar LC droplets in a single beam optical trap. While the bipolar droplets were trapped in a symmetrical manner, through its center, the radial droplets were trapped off-center, always about half way between the center and the edge of the droplet, see figure 4 . We have also observed continuous rotation of the bipolar droplets when the polarization of the trapping beam was set to certain 
orientation. In other polarization settings, it was possible to adjust the droplet into a fixed rotational angle. In case of radial droplets, in some polarization settings we have observed precession in all three dimensions, rather than the rotation. It was also possible to achieve continuous cyclic movement of the droplet, as well as several stable angles in which the droplet was trapped in a motionless manner.
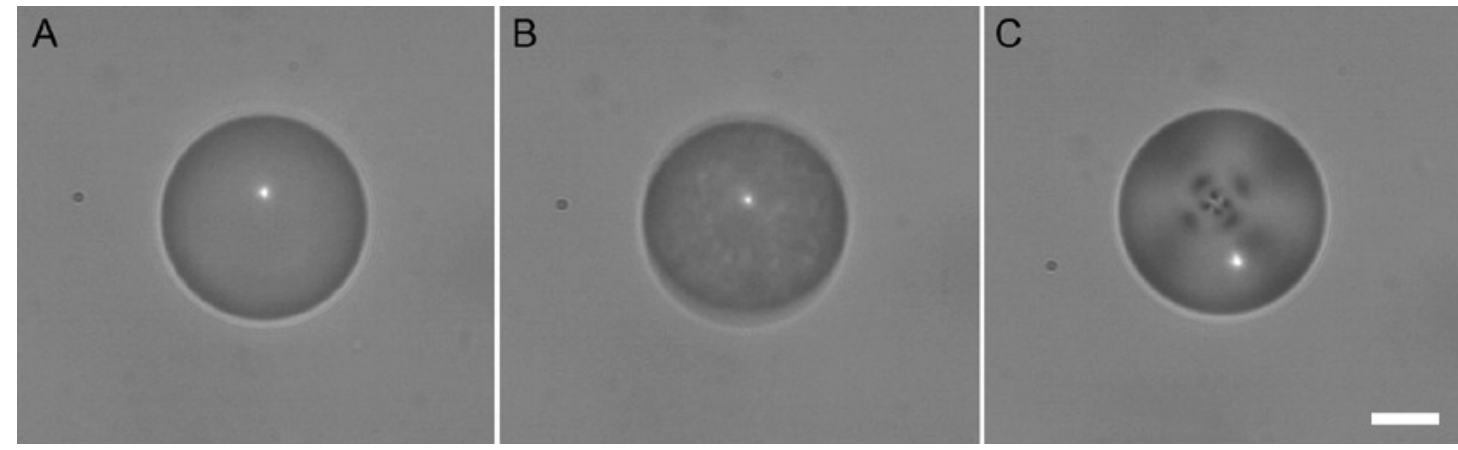

Figure 5: Phase transition of 5-CB LC droplet from isotropic (A) to intermediate (B) and to liquid crystalline state in radial configuration (C). The phase transition from LC phase to isotropic phase was achieved by heating the droplet by NIR radiation from the dual beam optical trap. When the dual beam laser power was reduced, the reverse transition from isotropic to LC state (in the picture) took its place. The transition appears the same in either direction. The bright spot near the center of the droplet is due to the auxiliary single beam trap. Scale-bar: $10 \mu \mathrm{m}$.

In a single beam trap, we were able to trap even the $\mathrm{LC}$ with low melting temperature (temperature of transition to isotropic phase), namely $5-\mathrm{CB}\left(\mathrm{m} . \mathrm{p} .35^{\circ} \mathrm{C}\right.$ ), without compromising their liquid crystalline structure. In a dual beam optical trap, the melting into isotropic phase was observed in 5-CB, see figure 5 . Therefore, for the dual beam trapping experiments with $\mathrm{LC}$ in their liquid crystalline phase, we employed mixtures of 5-CB with 8-OCB (m.p. $81^{\circ} \mathrm{C}$ ). Although it helps us to preserve the LC structure in higher temperatures, the temperature dependent behavior of such mixture is then probably rather complex: The pure 5-CB melts into isotropic phase at $35^{\circ} \mathrm{C}$, pure 8-OCB transitions from smectic-A phase to nematic phase at $61^{\circ} \mathrm{C}$, and finally melts to isotropic phase at $81^{\circ} \mathrm{C}$. All these transition temperatures are influenced by the mutual solubility of the two LCs and by the presence of dyes and surfactants.

We have observed the WGM lasing of the Nile Red doped 5-CB emulsion droplets in the dual beam trap, before and after the transition into the isotropic phase. We have detected a "jump" in the WGM emission wavelength. In the liquid crystalline phase, the emission is centered around $665 \mathrm{~nm}$, while in isotropic phase, the emission is around $640 \mathrm{~nm}$. No intermediate state was detected between the "switching" wavelengths, but shortly after the transition into the isotropic phase, we have detected a momentary "oscillation" state, displaying both the bands at $640 \mathrm{~nm}$ and $665 \mathrm{~nm}$. The spectra of the WGM emissions from all the three identified states are depicted in figure 6. In radial LC droplets, the result was the same whether the excitation was in- or off-axis. In case of bipolar LC droplets, on-axis excitation produced WGM and lasing, while off-axis excitation produced no WGM or lasing. In the isotropic phase, the placement of excitation beam did not play a role, WGM and lasing were observed in all cases. 


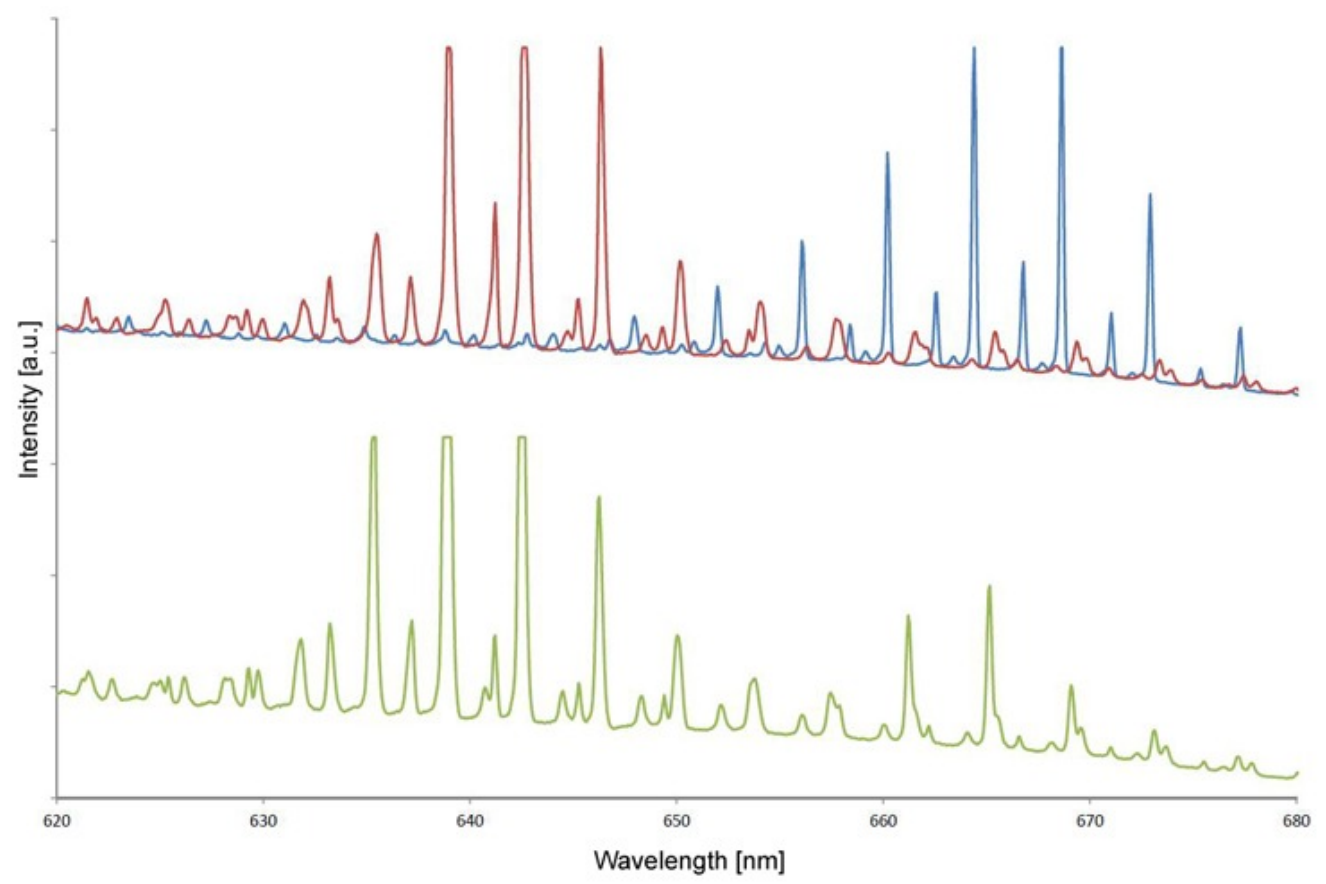

Figure 6: The spectra of WGM laser emission from LC droplet in LC phase (blue), in isotropic phase (red) and in an unidentified "oscillation" state several milliseconds after the LC to isotropic phase transition, which exhibits both emissions at once (green). The emissions in the two extreme states are centered around $640 \mathrm{~nm}$ (isotropic phase), and $665 \mathrm{~nm}$ (LC phase). In the LC phase, the droplet spontaneously attained a radial configuration.

In order to compare this result with the effect of simple ohmic heating of the emulsion droplets, we have used the heating chip, as described in the materials and methods section. (We have employed ohmic heating of the surrounding liquid and trapped the droplet in a single beam trap.) The observed results were the same as with heating by the dual beam trap, except for the bipolar droplets in the liquid crystalline phase, where they lased erratically, as the axis of the LC was not fixed, and the droplet was excited in different angles with respect to the molecular director. In contrast with these observations, when 5-CB was doped with DiI surfactant dye $\left(1,1^{\prime}\right.$ Dioctadecyl-3,3,3',3'-tetramethylindocarbocyanine perchlorate, Sigma-Aldrich), no "jump" in emission wavelength was observed at the phase transition whatsoever, the central emission wavelength being the same whether the LC was in liquid crystalline, or isotropic phase.

In the case of Nile Red doped LC mixture of 5-CB and 8-OCB, the dual beam trapping at increasing trapping power has yielded a shift in the WGM modes. When radial LC droplets were heated/stretched by the optical trap, both the in- and off-axis excitation has produced a blue shift, approx. $2.5 \mathrm{~nm}$ per $1 \mathrm{~W}$ of power increase in the optical trap, see figure 7. When bipolar LC droplets were used, a red shift of the same magnitude was detected for in-axis excitation, see figure 8 , but for off-axis excitation, there was no WGM at all. Curiously, when we tried to mimic these results by simple ohmic heating in the heating chip, we have detected no shift across a $10^{\circ} \mathrm{C}$ interval tested. Nevertheless, similar difference in temperature was indicated in the dual beam OT experiment, see [7]. The table summarizing all the experiments with heating/stretching of the various LC mixtures is depicted in figure 9. 


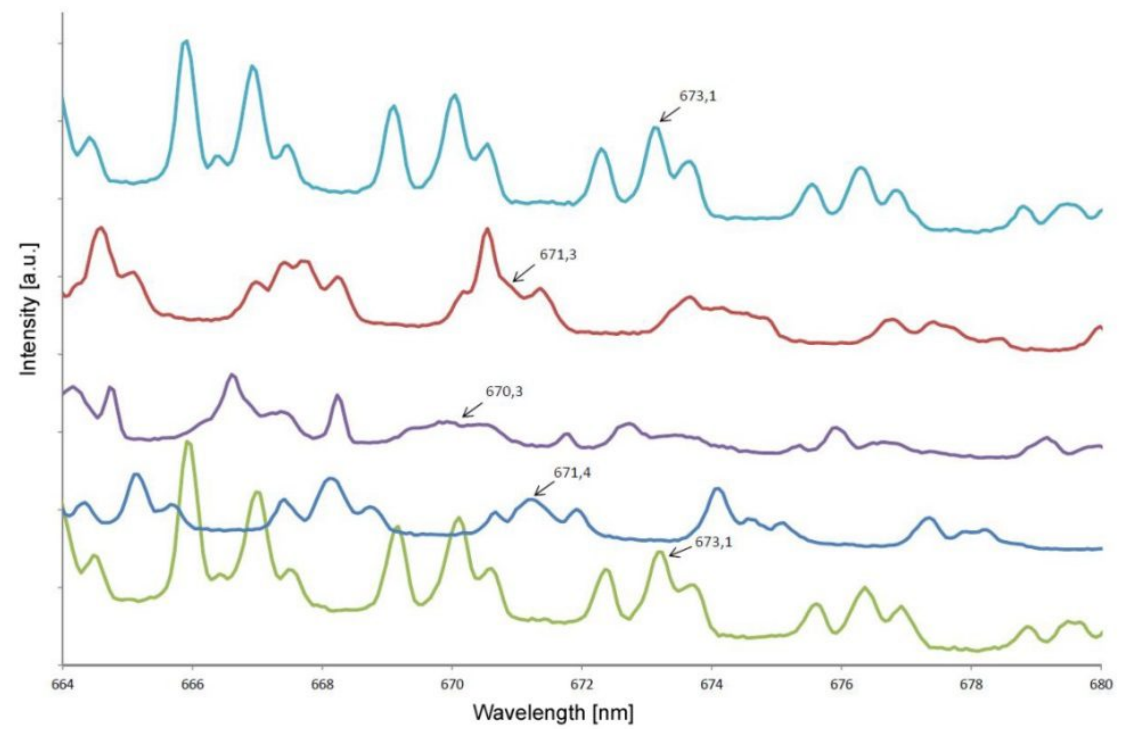

Figure 7: The spectra of WGM laser emission from radial LC droplet excited "in axis". From the top spectrum to the bottom spectrum, the droplet was heated and stretched with dual beam trap and released again. The middle spectrum shows the maximal heating and stretching. Blue shift is apparent with increase of heating and stretching power.

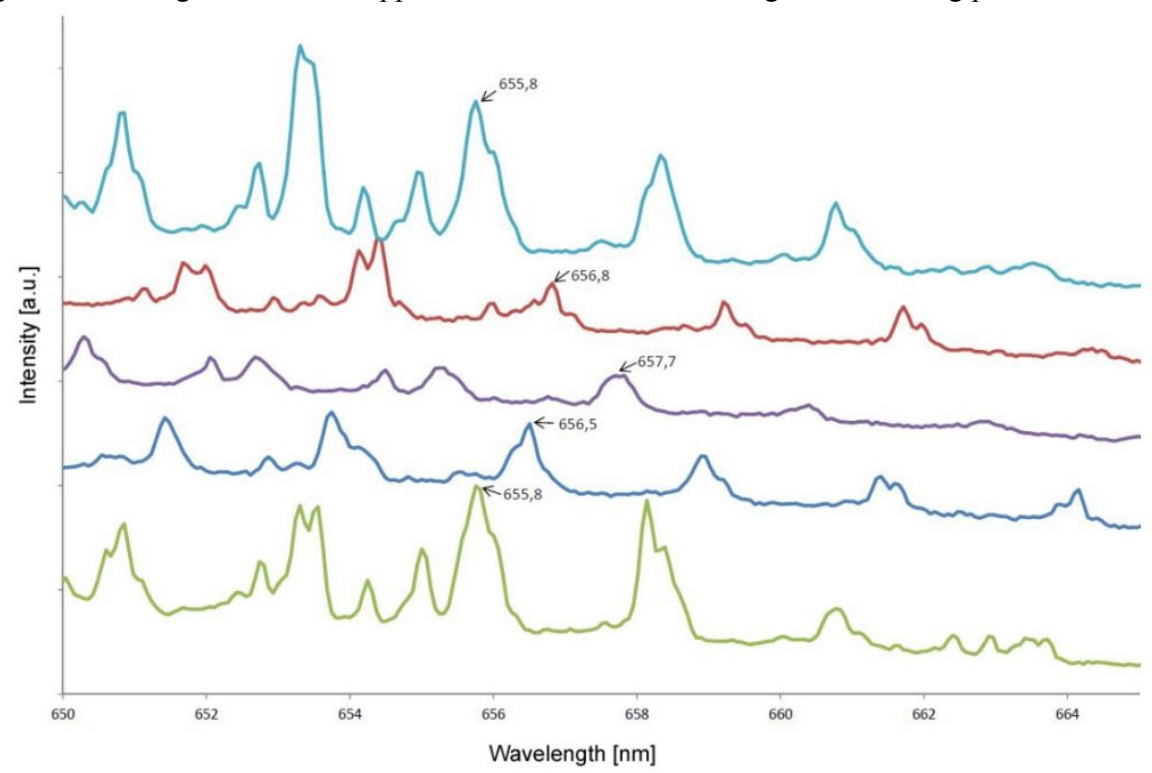

Figure 8: The spectra of WGM laser emission from bipolar LC droplet excited "in axis". From the top spectrum to the bottom spectrum, the droplet was heated and stretched with dual beam trap and released again. The middle spectrum shows the maximal heating and stretching. Red shift is apparent with increase of heating and stretching power. 


\begin{tabular}{|c|c|c|c|c|c|}
\hline & & LC arrangement & Ohmic heating +1 beam OT & 2 beam OT he & ating/stretching \\
\hline LC material & Fluorophore & & & In axis excitation & Off axis excitation \\
\hline $5 \mathrm{CB}+8 \mathrm{OCB} 1: 1$ & NR $0.1 \%$ & Radial - LC phase & Lasing - no shift $\left(10^{\circ} \mathrm{C}\right.$ sweep $)$ & Lasing - blue shift $(2.5 \mathrm{~nm} / 1 \mathrm{~W})$ & Lasing - blue shift $(2.5 \mathrm{~nm} / 1 \mathrm{~W})$ \\
\hline $5 \mathrm{CB}+80 \mathrm{CB} 1: 1$ & NR $0.1 \%$ & Bipolar - LC phase & Lasing - no shift $\left(10^{\circ} \mathrm{C}\right.$ sweep $)$ & Lasing - red shift $(2.5 \mathrm{~nm} / 1 \mathrm{~W})$ & No lasing - no shift \\
\hline $5 \mathrm{CB}$ & NR $0.1 \%$ & Radial - LC phase & Lasing & Lasing & Lasing \\
\hline $5 \mathrm{CB}$ & NR $0.1 \%$ & Isotropic phase & Lasing - blue shift $25 \mathrm{~nm}$ & Lasing - blue shift $25 \mathrm{~nm}$ & Lasing - blue shift $25 \mathrm{~nm}$ \\
\hline $5 \mathrm{CB}$ & NR $0.1 \%$ & Bipolar - LC phase & Lasing erratic (I/O axis) & Lasing & No lasing \\
\hline $5 \mathrm{CB}$ & NR $0.1 \%$ & Isotropic phase & Lasing - blue shift $25 \mathrm{~nm}$ & Lasing - blue shift $25 \mathrm{~nm}$ & Lasing - blue shift $25 \mathrm{~nm}$ \\
\hline $5 \mathrm{CB}$ & Dil $0.1 \%$ & Radial - LC phase & Lasing & NA & NA \\
\hline $5 \mathrm{CB}$ & Dil $0.1 \%$ & Isotropic phase & Lasing - no shift & NA & NA \\
\hline
\end{tabular}

Figure 9: A summary of the obtained results with various LC + fluorescent dye combinations, either subjected to ohmic heating by a resistor wire embedded in a microfluidic chip, or heated (and stretched) by the dual beam optical trap. In case of ohmic heating, the droplet was trapped only in the single beam trap, and therefore it was free to rotate around its axis. Polarization of the excitation beam was not established. In all cases, excitation beam was arranged to hit the edge of the droplet.

\section{CONCLUSIONS}

In our previous experiments [15], we have applied optical stretching and WGM excitation on fluorescent dye doped oil emulsion droplets. Our current experiment was devoted to examination of fluorescent dye doped liquid crystal emulsion droplets based WGM resonators. We have employed both ohmic heating and laser stretching and heating to tune the WGM lasing wavelength in the droplets. In our previous experiments we have achieved tuning efficiency of about $0.5 \mathrm{~nm}$ per $1 \mathrm{~W}$ of the stretching laser power. In our current experiment, the tuning efficiency with optical stretching reached $2.5 \mathrm{~nm} / \mathrm{W}$. We have also measured a blue shift of about $25 \mathrm{~nm}$ by heating the liquid crystals beyond the transition temperature.

\section{ACKNOWLEDGMENTS}

The research was supported by the Technology Agency of the Czech Republic (TA03010642), ASCR and TÜBITAK (Grant No. 111T059), Ministry of Education, Youth and Sports of the Czech Republic (LO1212, LD14069) together with the European Commission (ALISI No. CZ.1.05/2.1.00/01.0017).

\section{REFERENCES}

[1] Thorsen, T., Maerkl, S. J. and Quake, S. R., "Microfluidic large-scale integration," Science 298, 580-584 (2002).

[2] deMello, A. J., "Control and detection of chemical reactions in microfluidic systems, " Nature, 442, 394-402 (2006).

[3] Whitesides, G. M, "The origins and future of microfluidics," Nature 442, 368-373 (2006).

[4] Sery, M., Pilat, Z., Jonas, A., Jezek, J., Jakl, P., Zemanek, P., Samek, O., Nedbal, L., Trtilek, M., “Active sorting switch for biological objects," Proc. SPIE 7762, 776210:1-7 (2010).

[5] Terray, A., Oakey, J. and Marr, D. W. M., "Microfluidic control using colloidal devices," Science 296, 18411844 (2002).

[6] Terray, A., Oakey, J., and Marr, D. W. M., "Fabrication of linear colloidal structures for microfluidic applications," Appl. Phys. Lett. 81,1555-1557 (2002).

[7] Tanyeri, M., Perron, R. and Kennedy, I. M., "Lasing droplets in a microfabricated channel," Opt. lett. 32, 2529- 2531 (2007).

[8] Tkachenko, G. and Brasselet, E., “Optofluidic sorting of material chirality by chiral light,” Nat. comm. 5:3577, 1-7 (2014).

[9] Humar, M. and Musevic, I., "Surfactant sensing based on whispering-gallery-mode lasing in liquid-crystal microdroplets," Opt. express 19, 19836-19844 (2011).

[10] Humar, M. and Musevic, I., "3D microlasers from self-assembled cholesteric liquid-crystal microdroplets ," Opt. express 26, 26995- 27003 (2010). 
[11] Humar, M., Ravnik, M., Pajk, S., and Musevic, I., "Electrically tunable liquid crystal optical microresonators," Nat. photonics 3, 595-600 (2009).

[12] Tang, S. K. Y., Li, Z., Abate, A. R., Agresti, J. J., Weitz, D. A., Psaltis, D., and Whitesides, G. M., “A multi-color fast-switching microfluidic droplet dye laser," Lab Chip 9, 2767-2771 (2009).

[13] Tang, S. K. Y., Derda, R., Quan, Q., Loncar, M., and Whitesides, G. M., "Continuously tunable microdroplet-laser in a microfluidic channel," Opt. Express 19, 2204-2215 (2011).

[14] Donato, M. G., Hernandez, J., Mazzulla, A., Provenzano, C., Saija, R., Sayed, R., Vasi, S., Magazzu, A., Pagliusi, P., Bartolino, R., Gucciardi, P.G., Marago, O.M., and Cipparrone, G., "Polarization-dependent optomechanics mediated by chiral microresonators," Nat. comm. 5:3656, 1-7 (2014).

[15] Aas, M., Jonas, A., Kiraz, A., Brzobohaty, O., Jezek, J., Pilat, Z., Zemanek, P., "Spectral tuning of lasing emission from optofluidic droplet microlasers using optical stretching," Optics Express 21, 21381-21394 (2013). 